\section{Meandering winds precede heat spells}

Although the deadly heatwaves that afflict the United States arise from chaotic forces, they could be predicted up to three weeks before they hit.

As yet, heatwaves cannot be foreseen until about ten days in advance. However, in a 12,000-year computer simulation of general atmospheric circulation, Haiyan Teng at the National Center for Atmospheric Research in Boulder, Colorado, and her colleagues observed patterns in the meanderings of high-altitude winds that tended to precede North American heatwaves by 15-20 days.

In the model, large-scale circulation anomalies that increased the risk of heatwaves were not linked to other commonly used indicators, such as monsoons or sea surface temperatures. Monitoring mid-latitude atmospheric dynamics could allow more accurate and longer-term forecasts, the authors say.

Nature Geosci. http://doi.org/ pnt (2013)

For a longer story on this research, see go.nature.com/fjsuha

\section{PLANETARY SCIENCE}

\section{Why Martian} craters are flat

When meteorites pummelled a young Mars, they may have obliterated enough of its crust to allow the planet's mantle to well up and trigger volcano-like activity across the red planet.

A team led by Christopher Edwards of the California Institute of Technology in Pasadena looked at 2,800 ancient Martian craters with flat floors, thought to be made of sediment layers. But data from orbiting satellites showed that materials in the crater were too rocky for this to be true. Other observations, such as the materials' mineral composition, were also incompatible with sediment.
Instead, the authors propose, meteorite impacts that fractured the planet's crust provided paths for molten rock to flow up and flood the crater floors. At a time when Earth's early oceans supported life, the surface of Mars may have been marked by magma.

Icarus http://doi.org/pd2 (2013)

\section{NEUROSCIENCE \\ Rats propelled by electric pulses}

Deep-brain stimulation can help rats with injured spinal cords to walk. After severing a large portion of neural fibres in rats' spines, researchers led by Lukas Bachmann at the University of Zurich in Switzerland applied electrical pulses to a brainstem region that controls locomotion.

This allowed rats with only about $20 \%$ of intact neural fibres to walk almost normally, even though similar injuries in rats and humans cause severe impairments in walking. Even rats with less than $10 \%$ of these fibres and completely paralysed hindlimbs regained some movement when swimming.

Although more work is necessary to evaluate the approach's therapeutic potential, tapping into a person's existing motor command circuitry in the brain may provide more control over voluntary movements than do other approaches that stimulate the spinal cord itself.

Sci. Transl. Med. 5, 208ra146 (2013)

\section{EVOLUTION}

\section{Human ancestor had small thumbs}

Fossil analysis reveals that an ancestor of modern humans would have made a terrible hitch-hiker.

Past reconstructions of the hands of the hominin Australopithecus afarensis assigned scattered bones to individuals and single fingers.

\title{
Tau tangles exposed
}

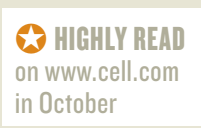

Tangles of the tau protein are found in the brains of people who have died with Alzheimer's disease, but establishing techniques to see the protein in living

people has been difficult.

A team led by Makoto Higuchi at the National Institute of Radiological Sciences in Chiba, Japan, developed a class of radioactive molecule that can be used to image live brains.

The researchers first confirmed that the molecules bound to tau using brain slices from patients with Alzheimer's disease and in mutant mice with tau deposits in their brains. The team then injected the imaging molecules into human participants. In three people with probable disease, imaging using positron emission tomography showed the molecules binding in brain

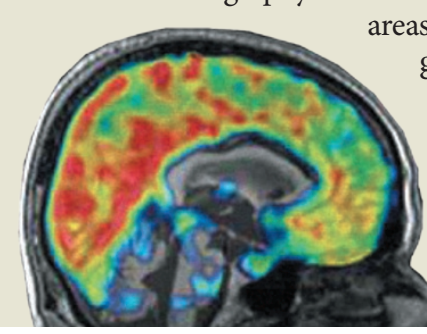

Campbell Rolian at the University of Calgary, Canada, and Adam Gordon at the State University of New York at Albany reanalysed an assembly of $A$. afarensis bones to better account for uncertainties in the fossil record.

Their results suggest that the hominin had shorter thumbs than was thought, with proportions more closely resembling those of gorillas. Although A. afarensis may have been able to bring the tips of its fingers and thumbs together, its thumbs were not long enough for the precision grip that later hominins used to craft stone tools.

Am. J. Phys. Anthropol. 152, 393-406 (2013)

VIROLOAY

\section{Not all dormant HIV is defective}

Viral 'sleeper agents' in human genomes could make HIV even harder to cure than expected.

Dormant HIV sequences called proviruses embed themselves in the DNA of immune-system cells, thwarting efforts to eliminate HIV infection. However, in laboratory tests, less than $1 \%$ of proviruses could be reactivated - the rest were thought to be so mutated that they are unable to awaken and spread.

Now, Robert Siliciano of Johns Hopkins University in Baltimore, Maryland, and his colleagues show that in people who have been treated for HIV, the proportion of potentially active proviruses is higher than assumed. The researchers sequenced DNA of 213 inactive proviruses from people with HIV and found that around $12 \%$ of proviruses still had intact genomes. That makes the reservoir of latent virus some 60 times larger than previous estimates.

Cell 155, 540-551 (2013)

\section{DNATURE.COM}

For the latest research published by Naturevisit:

www.nature.com/latestresearch 